Moroccan J. of Pure and Appl. Anal. (MJPAA)

Volume 7(3), 2021, Pages 461-469

ISSN: Online 2351-8227 - Print 2605-6364

DOI: $10.2478 / \mathrm{mjpaa}-2021-0031$

\title{
Jacobson's Lemma in the ring of quaternionic linear operators
}

\author{
El Hassan Benabdi ${ }^{1}$ and Mohamed BarraA ${ }^{2}$
}

\begin{abstract}
Aвstract. In the present paper, we study the Jacobson's Lemma in the unital ring of all bounded right linear operators $\mathcal{B}_{R}(X)$ acting on a two-sided quaternionic Banach space $X$. In particular, let $A, B \in \mathcal{B}_{R}(X)$ and let $q \in \mathbb{H} \backslash\{0\}$, we prove that $w(A B) \backslash\{0\}=w(B A) \backslash\{0\}$ where $w$ belongs to the spherical spectrum, the spherical approximate point spectrum, the right spherical spectrum, the left spherical spectrum, the spherical point spectrum, the spherical residual spectrum and the spherical continuous spectrum. We also prove that the range of $(A B)^{2}-2 \operatorname{Re}(q) A B+|q|^{2} I$ is closed if and only if $(B A)^{2}-2 \operatorname{Re}(q) B A+|q|^{2} I$ has closed range. Finally, we show that $(A B)^{2}-2 \operatorname{Re}(q) A B+|q|^{2} I$ is Drazin invertible if and only if $(B A)^{2}-2 \operatorname{Re}(q) B A+|q|^{2} I$ is.
\end{abstract}

Mathematics Subject Classification (2020). 46S10, 47S10, 47A10.

Key words and phrases. Quaternionic Banach space, Spherical spectrum, Quaternionic Banach algebra.

\section{Introduction and preliminaries}

Jacobson's Lemma states that for any ring $\mathcal{R}$ with unit $1,1-a b$ is invertible if and only if $1-b a$ is. Moreover, $(1-b a)^{-1}=1+b(1-a b)^{-1} a$. If $\mathcal{R}$ is a complex Banach algebra, then the Jacobson's Lemma implies that for any $\lambda \in \mathbb{C} \backslash\{0\}, \lambda 1-a b$ is invertible if and only if $\lambda 1-b a$ is (see, for instance, [2, Lemma 3.1.2]). In other words, $\sigma(a b) \backslash\{0\}=\sigma(b a) \backslash\{0\}$, where $\sigma(a)$ denotes the spectrum of $a$.

Barnes [3] proved that if $A$ and $B$ are bounded linear operators on a complex Banach space,

Received June 18, 2020 - Accepted: April 13, 2021.

(C) The Author(s) 2021. This article is published with open access by Sidi Mohamed Ben Abdallah University.

1,2Department of Mathematics, Faculty of Sciences Semlalia, Cadi Ayyad University, Marrakesh, Morocco. e-mail ${ }^{1}$ : elhassan.benabdi@gmail.com (Corresponding Author).

e-mail²: barraa@hotmail.com. 
then $A B$ and $B A$ have the same spectrum, approximate point spectrum, point spectrum, residual spectrum and continuous spectrum up to zero. Moreover, the range of $I-A B$ is closed if and only if so is the range of $I-B A$.

Cvetković-Ilić and Harte [6] extended Jacobson's Lemma to the Drazin invertibility. Furthermore, they proved that $1-a b$ and $1-b a$ always have the same Drazin index. Lam and Nielsen [10] gave explicit formula relating the Drazin inverses of $1-a b$ and $1-b a$, see also [11, 14].

We observe that in the case in which $\mathcal{R}$ is the algebra of quaternions, [2, Lemma 3.1.2] does not hold. For instance, let $\mathrm{i}, \mathrm{j}, \mathrm{k} \in \mathcal{R}$ such that $\mathrm{i}^{2}=\mathrm{j}^{2}=\mathrm{k}^{2}=\mathrm{ijk}=-1$. Then $\mathrm{k} 1-\mathrm{ji}$ is invertible but $\mathrm{k} 1-\mathrm{ij}$ is not. So, one may ask is there any version of [2, Lemma 3.1.2] for bounded right linear operators on two-sided quaternionic Banach spaces.

We finish this section with basic definitions and notations of quaternions, quaternionic Banach spaces and spherical spectrum. In Section 2 we extend Jacobson's Lemma to the set of all bounded right linear operators acting on a two-sided quaternionic Banach space. In particular, if $A$ and $B$ are such operators, then $A B$ and $B A$ have the same spherical spectrum, spherical approximate point spectrum, right spherical spectrum, left spherical spectrum, spherical point spectrum, spherical residual spectrum and spherical continuous spectrum up to zero. Further, we give analogues of Jacobson's Lemma for many right linear operator properties.

Quaternions. Let $\mathbb{H}$ be the set of all elements, called quaternions, of the form $q=a+b \mathbf{i}+c \mathbf{j}+$ $d \mathrm{k} ; a, b, c, d \in \mathbb{R}$, where $\mathrm{i}, \mathrm{j}$ and $\mathrm{k}$ are quaternion units satisfying:

$$
\mathrm{i}^{2}=\mathrm{j}^{2}=\mathrm{k}^{2}=\mathrm{ijk}=-1 \text {. }
$$

Then $\mathbb{H}$ is a non-commutative algebra with the addition defined same as in $\mathbb{C}$ and multiplication given by Equations (1.1). For a given $q \in \mathbb{H}$, we define the real part of $q, \operatorname{Re}(q):=a$ and the imaginary part of $q, \operatorname{Im}(q):=b \mathbf{i}+c j+d \mathrm{k}$. The conjugate and the modulus of $q$ are given respectively by

$$
\bar{q}=a-b \mathbf{i}-c \mathbf{j}-d \mathrm{k},|q|=\sqrt{q \bar{q}}=\sqrt{a^{2}+b^{2}+c^{2}+d^{2}} .
$$

For the standard results of the algebra of quaternions $\mathbb{H}$, the reader is referred, for instance, to $[4,8]$ and the references therein.

\section{Quaternionc Banach spaces.}

Definition 1.1. Let $(X,+)$ be an abelian group. $X$ is a left (resp. right) quaternionic vector space if it is endowed with a left (resp. right) quaternionic multiplication $(\mathbb{H}, X) \rightarrow X,(q, x) \mapsto q x$ (resp. $(X, \mathbb{H}) \rightarrow X,(x, q) \mapsto x q)$ such that for all $x, y \in X$ and all $p, q \in \mathbb{H}$, we have:

(i) $q(x+y)=q x+q y(\operatorname{resp} .(x+y) q=x q+y q)$;

(ii) $(p+q) x=p x+q x(\operatorname{res} p \cdot x(p+q)=x p+x q)$;

(iii) $q(p x)=(q p) x(\operatorname{resp} .(x p) q=x(p q))$;

(iv) $1 x=x(\operatorname{resp} . x 1=x)$.

$X$ is a two-sided quaternionic vector space if it is both left and right quaternionic vector space and such that for all $x \in X, p, q \in \mathbb{H}$ and all $r \in \mathbb{R}$, we have:

$$
q(x p)=(q x) p \text { and } r x=x r .
$$


Definition 1.2. Let $X$ be a left (resp. right) quaternionic vector space. A function $\|\cdot\|: X \rightarrow[0 ;+\infty)$ is called a norm on $X$, if it satisfies

(i) $\|x\|=0$ if and only if $x=0$;

(ii) $\|q x\|=|q|\|x\|$ (resp. $\|x q\|=\|x\||q|$ ) for all $x \in X$ and all $q \in \mathbb{H}$;

(iii) $\|x+y\| \leq\|x\|+\|y\|$ for all $x, y \in X$.

If $X$ is complete with respect to the metric induced by $\|\cdot\|$, we call $X$ a left (resp. right) quaternionic Banach space.

A two-sided quaternionic vector space $X$ is a two-sided quaternionic Banach space if it is both left and right quaternionic Banach space.

Definition 1.3. Let $X$ and $Y$ be two right quaternionic Banach spaces. A right linear operator of $X$ into $Y$ is a map $T: X \rightarrow Y$ such that:

$$
T(x q+y)=(T x) q+T y \text { for all } x, y \in X \text { and all } q \in \mathbb{H} \text {. }
$$

A right linear operator $T$ of $X$ into $Y$ is called bounded if

$$
\|T\|:=\sup \{\|T x\|: x \in X,\|x\|=1\}<\infty .
$$

The set of all right linear bounded operators of $X$ into $Y$ is denoted by $\mathcal{B}_{R}(X, Y)$. Set $\mathcal{B}_{R}(X):=$ $\mathcal{B}_{R}(X, X)$ for short: the set of all right linear bounded operators on $X$.

Definition 1.4. A two-sided quaternionic Banach algebra is a two-sided quaternionic Banach space $\mathcal{A}$ that is endowed with a product $\mathcal{A} \times \mathcal{A} \rightarrow \mathcal{A}$ such that:

(i) The product is associative and distributive over the sum in $\mathcal{A}$;

(ii) one has $(q x) y=q(x y)$ and $x(y q)=(x y) q$ for all $x, y \in \mathcal{A}$ and all $q \in \mathbb{H}$;

(iii) one has $\|x y\| \leq\|x\|\|y\|$ for all $x, y \in \mathcal{A}$.

If in addition there exists $e \in \mathcal{A}$ such that $\|e\|=1$ and ex $=x e=x$ for all $x \in \mathcal{A}$, then $\mathcal{A}$ is called $a$ two-sided quaternionic Banach algebra with unit.

Here are some examples of two-sided quaternionic Banach algebras.

1) The algebra of quaternions $\mathbb{H}$ is a two-sided quaternionic Banach algebra with unit.

2) The class of all $n \times n$ (for $n \in \mathbb{N}$ ) matrices over $\mathbb{H}$ equipped with the natural structure is a two-sided quaternionic Banach algebra with unit.

3) Let $X$ be a two-sided quaternionic Banach space. Then $\mathcal{B}_{R}(X)$ is a two-sided quaternionic Banach algebra; the sum is the usual pointwise sum, the product is the composition and the quaternionic multiplications are defined by setting

$$
(q T) x:=q(T x),(T q) x:=T(q x) \quad \text { for all } T \in \mathcal{B}_{R}(X), x \in X \text { and all } q \in \mathbb{H} .
$$

For more material about quaternionic operators and other information on the basic theory of quaternionic Banach spaces, we refer the reader to [4, 5] and [8].

Spherical spectrum. Colombo, Sabadini and Struppa [5] extended the definitions of the spectrum and resolvent in quaternionic Banach spaces as follows.

Definition 1.5. Let $X$ be a two-sided quaternionic Banach space and let $T \in \mathcal{B}_{R}(X)$. For $q \in \mathbb{H}$, we set

$$
Q_{q}(T):=T^{2}-2 \operatorname{Re}(q) T+|q|^{2} I
$$


Where $I$ is the identity operator on $X$. We define the spherical resolvent set $\rho_{S}(T)$ of $T$ as

$$
\rho_{S}(T):=\left\{q \in \mathbb{H}: Q_{q}(T) \text { is invertible in } \mathcal{B}_{R}(X)\right\},
$$

and we define the spherical spectrum $\sigma_{S}(T)$ of $T$ as

$$
\sigma_{S}(T):=\mathbb{H} \backslash \rho_{S}(T) .
$$

The right spherical spectrum $\sigma_{S r}(T)$ and the left spherical spectrum $\sigma_{S l}(T)$ are defined respectively as

$$
\begin{aligned}
& \sigma_{S r}(T):=\left\{q \in \mathbb{H}: Q_{q}(T) \text { is not right invertible in } \mathcal{B}_{R}(X)\right\} ; \\
& \sigma_{S l}(T):=\left\{q \in \mathbb{H}: Q_{q}(T) \text { is not left invertible in } \mathcal{B}_{R}(X)\right\} .
\end{aligned}
$$

The spherical approximate point spectrum is defined by

$$
\sigma_{a S}(T):=\left\{q \in \mathbb{H}: \text { there is a sequence }\left\{x_{n}\right\} \text { of unit vectors in } X \text { such that }\left\|Q_{q}(T) x_{n}\right\| \rightarrow 0\right\} \text {. }
$$

For $T \in \mathcal{B}_{R}(X)$, let $\mathcal{N}(T)$ denote the null space of $T$, and let $\mathcal{R}(T)$ denote the range of $T$. The spectrum $\sigma_{S}(T)$ decomposes into three disjoint subsets as follows:

(i) The spherical point spectrum of $T$ :

$$
\sigma_{p S}(T):=\left\{q \in \mathbb{H}: \mathcal{N}\left(Q_{q}(T)\right) \neq\{0\}\right\} .
$$

(i) The spherical residual spectrum of $T$ :

$$
\sigma_{r S}(T):=\left\{q \in \mathbb{H}: \mathcal{N}\left(Q_{q}(T)\right)=\{0\} \text { and } \overline{\mathcal{R}\left(Q_{q}(T)\right)} \neq X\right\} .
$$

(iii) The spherical continuous spectrum of $T$ :

$$
\sigma_{c S}(T):=\left\{q \in \mathbb{H}: \mathcal{N}\left(Q_{q}(T)\right)=\{0\}, \overline{\mathcal{R}\left(Q_{q}(T)\right)}=X \text { and } \mathcal{R}\left(Q_{q}(T)\right) \neq X\right\} .
$$

If $T x=x q$ for some $q \in \mathbb{H}$ and $x \in X \backslash\{0\}$, then $x$ is called eigenvector of $T$ with eigenvalue q. The next proposition is well-known, see [4, Proposition 3.1.9], see also [8, Proposition 4.5].

Proposition 1.1. Let $X$ be a two-sided quaternionic Banach space and let $T \in \mathcal{B}_{R}(X)$. Then $\mathcal{N}\left(Q_{q}(T)\right) \neq\{0\}$ if and only if $q$ is an eigenvalue of $T$. Hence $\sigma_{p S}(T)$ coincides with the set of all eigenvalues of $T$.

\section{Main results}

In the following $X$ will be a fixed two-sided quaternionic Banach space and $\mathcal{A}$ will be a two-sided quaternionic Banach algebra with unit $e$. For convenience, we define the element $\delta_{a, b}:=(a b)^{2}-2 \operatorname{Re}(q) a b+|q|^{2} e$ for all $a, b \in \mathcal{A}$ and $q \in \mathbb{H}$.

Lemma 2.1 (Jacobson). Let $a, b \in \mathcal{A}$ and let $q \in \mathbb{H} \backslash\{0\}$. Then $\delta_{a, b}$ is invertible (resp. left invertible, right invertible) in $\mathcal{A}$ if and only if $\delta_{b, a}$ is invertible (resp. left invertible, right invertible) in $\mathcal{A}$.

Proof. Suppose that $\delta_{a, b}$ has a left (resp. right) inverse in $\mathcal{A}$. Then there is $c \in \mathcal{A}($ resp. $d \in \mathcal{A})$ such that $c \delta_{a, b}=e\left(\right.$ resp. $\left.\delta_{a, b} d=e\right)$. Since $\delta_{a, b} a=a \delta_{b, a}$ and $b \delta_{a, b}=\delta_{b, a} b$,

$$
\begin{aligned}
(2 \operatorname{Re}(q) b c a-b a b c a+e) \delta_{b, a} & =|q|^{2} e ; \\
\delta_{b, a}(2 \operatorname{Re}(q) b d a-b d a b a+e) & =|q|^{2} e .
\end{aligned}
$$

Then $\delta_{b, a}$ is left (resp. right) invertible in $\mathcal{A}$. 
If $\delta_{a, b}$ is invertible, then

$$
\left(\delta_{b, a}\right)^{-1}=\frac{1}{|q|^{2}}\left(2 \operatorname{Re}(q) b\left(\delta_{a, b}\right)^{-1} a-b\left(\delta_{a, b}\right)^{-1} a b a+e\right) .
$$

Theorem 2.1. Let $A, B \in \mathcal{B}_{R}(X)$. Then

(i) $\sigma_{S}(A B) \backslash\{0\}=\sigma_{S}(B A) \backslash\{0\}$;

(ii) $\sigma_{S r}(A B) \backslash\{0\}=\sigma_{S r}(B A) \backslash\{0\}$;

(iii) $\sigma_{S l}(A B) \backslash\{0\}=\sigma_{S l}(B A) \backslash\{0\}$;

(iv) $\sigma_{p S}(A B) \backslash\{0\}=\sigma_{p S}(B A) \backslash\{0\}$;

(v) $\sigma_{a S}(A B) \backslash\{0\}=\sigma_{a S}(B A) \backslash\{0\}$;

(vi) $\sigma_{r S}(A B) \backslash\{0\}=\sigma_{r S}(B A) \backslash\{0\}$;

(vii) $\sigma_{c S}(A B) \backslash\{0\}=\sigma_{c S}(B A) \backslash\{0\}$.

Proof. (i), (ii) and (iii) follow from Lemma 2.1.

(iv) Let $q \in \sigma_{p S}(A B) \backslash\{0\}$ then by Proposition 1.1, there exists an $x \in X \backslash\{0\}$ such that $A B x=x q$. Hence $B x \neq 0$ and therefore

$$
B(A B x)=B A(B x)=(B x) q .
$$

This implies that $\sigma_{p S}(A B) \backslash\{0\} \subseteq \sigma_{p S}(B A) \backslash\{0\}$. The second inclusion can be proved similarly. (v) Let $q \in \sigma_{a S}(A B) \backslash\{0\}$ then there exists a sequence $\left\{x_{n}\right\} \subset X$ such that $\left\|x_{n}\right\|=1$ for all $n \in \mathbb{N}$ and such that $\left(\delta_{A, B}\right) x_{n} \underset{n \rightarrow+\infty}{\longrightarrow} 0$. Hence $\left\{B x_{n}\right\}$ does not converge to 0 . Moreover,

$$
\delta_{B, A} B x_{n}=B \delta_{A, B} x_{n} \underset{n \rightarrow+\infty}{\longrightarrow} 0 .
$$

Thus $\sigma_{a S}(A B) \backslash\{0\} \subseteq \sigma_{a S}(B A) \backslash\{0\}$ and similarly one can show that $\sigma_{a S}(A B) \backslash\{0\} \supseteq \sigma_{a S}(B A) \backslash$ $\{0\}$.

(vi) Let $q \in \sigma_{r S}(A B) \backslash\{0\}$ and assume that $\overline{\mathcal{R}\left(\delta_{B, A}\right)}=X$. Let $x \in X$, then there are two sequences $\left\{x_{n}\right\} \subset X$ and $\left\{y_{n}\right\} \subset X$ such that

$$
\begin{aligned}
& \delta_{B, A} x_{n} \underset{n \rightarrow+\infty}{\longrightarrow} B x \\
& \delta_{B, A} y_{n} \underset{n \rightarrow+\infty}{\longrightarrow} B A B x .
\end{aligned}
$$

Hence

$$
\delta_{A, B}\left(2 \operatorname{Re}(q) A x_{n}-A y_{n}+x\right) \underset{n \rightarrow+\infty}{\longrightarrow}|q|^{2} x
$$

and so $\delta_{A, B}$ has dense range, which is a contradiction with the assumption that $q \in \sigma_{r S}(A B) \backslash$ $\{0\}$. Thus

$$
\overline{\mathcal{R}\left(\delta_{B, A}\right)} \neq X
$$

and so $\sigma_{r S}(A B) \backslash\{0\} \subseteq \sigma_{r S}(B A) \backslash\{0\}$. The inclusion $\sigma_{r S}(A B) \backslash\{0\} \supseteq \sigma_{r S}(B A) \backslash\{0\}$ is proved in a similar way.

(vii) Since $\left\{\sigma_{p S}(A B), \sigma_{r S}(A B), \sigma_{c S}(A B)\right\}$ is a partition of $\sigma_{S}(A B)$,

$$
\sigma_{c S}(A B) \backslash\{0\}=\sigma_{S}(A B) \backslash\left[\sigma_{p S}(A B) \cup \sigma_{r S}(A B) \cup\{0\}\right] .
$$

By (i), (iv) and (vi) it follows that

$$
\sigma_{S}(A B) \backslash\left[\sigma_{p S}(A B) \cup \sigma_{r S}(A B) \cup\{0\}\right]=\sigma_{S}(B A) \backslash\left[\sigma_{p S}(B A) \cup \sigma_{r S}(B A) \cup\{0\}\right]=\sigma_{c S}(B A) \backslash\{0\} .
$$


Finally, $\sigma_{c S}(A B) \backslash\{0\}=\sigma_{c S}(B A) \backslash\{0\}$.

As in the complex case, the reduced minimum modulus of $T \in \mathcal{B}_{R}(X)$ is defined by

$$
\gamma(T):=\inf \{\|T x\|: x \in X \text { and } \operatorname{dist}(x, \mathcal{N}(T))=1\} .
$$

Formally we set $\gamma(0)=\infty$.

We omit the proof of the following theorem, since it works exactly as in the complex case (see, e.g., [12, p. 99]).

Theorem 2.2 (The open mapping theorem). Let $X_{1}$ and $X_{2}$ be two right quaternionic Banach spaces. Let $T$ be a right linear operator from $X_{1}$ to $X_{2}$. The following conditions are equivalent:

(i) $T$ is onto;

(ii) there exists a positive constant $k$ such that $k \mathbb{B}_{X_{2}} \subseteq T \mathbb{B}_{X_{1}}$.

Where $\mathbb{B}_{X_{1}}$ and $\mathbb{B}_{X_{2}}$ are respectively the unit open balls of $X_{1}$ and $X_{2}$.

Corollary 2.1. Let $X_{1}$ and $X_{2}$ be two right quaternionic Banach spaces. If an operator $T \in \mathcal{B}_{R}\left(X_{1}, X_{2}\right)$ is one-to-one and onto, then $T^{-1}$ is bounded.

Theorem 2.3. Let $T \in \mathcal{B}_{R}(X)$. Then $\mathcal{R}(T)$ is closed if and only if $\gamma(T)>0$.

Proof. Clearly $X / \mathcal{N}(T)$ with the natural structure inherited from $X$ is a right quaternionic vector space. When equipped with the norm $\|x+\mathcal{N}(T)\|=\operatorname{dist}(x, \mathcal{N}(T)), X / \mathcal{N}(T)$ is a right quaternionic Banach space. Define the operator

$$
T_{0}: \begin{array}{ccc}
X / \mathcal{N}(T) & \longrightarrow & \overline{\mathcal{R}(T)} \\
T_{0}(x+\mathcal{N}(T)) & \longmapsto T x .
\end{array}
$$

If $\mathcal{R}(T)$ is closed. Then $T_{0}$ is bijective. By Corollary 2.1, $T_{0}^{-1}$ is bounded, and so $\left\|T_{0}^{-1} T x\right\| \leq$ $\left\|T_{0}^{-1}\right\|\|T x\|$. Thus $\gamma(T)=\frac{1}{\left\|T_{0}^{-1}\right\|}>0$.

If $\gamma(T)>0$. Then $\gamma(T)\|x+\mathcal{N}(T)\| \leq\|T x\|$. Thus $\mathcal{R}(T)$ is complete and therefore closed in $X$.

Theorem 2.4. Let $A, B \in \mathcal{B}_{R}(X)$ and let $q \in \mathbb{H} \backslash\{0\}$. Then $\mathcal{R}\left(\delta_{A, B}\right)$ is closed if and only if $\mathcal{R}\left(\delta_{B, A}\right)$ is closed.

Proof. Assume that $\mathcal{R}\left(\delta_{A, B}\right)$ is closed. Then $\alpha:=\gamma\left(\delta_{A, B}\right)>0$. Let $s:=\frac{1}{|q|^{2}}, t:=\frac{2 \operatorname{Re}(q)}{|q|^{2}}$ and let $x \in X$. Hence

$$
\left\|\delta_{A, B} A x\right\|=\left\|A \delta_{B, A} x\right\| \leq\|A\|\left\|\delta_{B, A} x\right\| .
$$

Since

$$
\begin{gathered}
\alpha \inf \left\{\|A x-y\|: y \in \mathcal{N}\left(\delta_{A, B}\right)\right\} \leq\left\|\delta_{A, B} A x\right\|, \\
\alpha \inf \left\{\|(t B-s B A B)(A x-y)\|: y \in \mathcal{N}\left(\delta_{A, B}\right)\right\} \leq\|t B-s B A B\|\|A\|\left\|\delta_{B, A} x\right\| .
\end{gathered}
$$

It is clear that $(t B-s B A B) y \in \mathcal{N}\left(\delta_{B, A}\right)$ for all $y \in \mathcal{N}\left(\delta_{A, B}\right)$. Thus

$$
\alpha \inf \left\{\|(t B-s B A B) A x-y\|: y \in \mathcal{N}\left(\delta_{B, A}\right)\right\} \leq\|t B-s B A B\|\|A\|\left\|\delta_{B, A} x\right\| .
$$


Therefore

$$
\alpha \inf \left\{\|x-y\|: y \in \mathcal{N}\left(\delta_{B, A}\right)\right\} \leq\|t B-s B A B\|\|A\|\left\|\delta_{B, A} x\right\|+\alpha\left\|s(B A)^{2} x-t B A x+x\right\|,
$$

and so

$$
\alpha \inf \left\{\|x-y\|: y \in \mathcal{N}\left(\delta_{B, A}\right)\right\} \leq\left(\|t B-s B A B\|\|A\|+\frac{\alpha}{|q|^{2}}\right)\left\|\delta_{B, A} x\right\| .
$$

It follows that $\gamma\left(\delta_{B, A}\right)>0$, then by Theorem 2.3, $\mathcal{R}\left(\delta_{B, A}\right)$ is closed.

Given $T \in \mathcal{B}_{R}(X)$. The ascent of $T \in \mathcal{B}_{R}(X)$ is defined as $\operatorname{asc}(T)=\inf \left\{n: \mathcal{N}\left(T^{n}\right)=\right.$ $\left.\mathcal{N}\left(T^{n+1}\right)\right\}$, and the descent of $T$ is defined as $d s c(T)=\inf \left\{n: \mathcal{R}\left(T^{n}\right)=\mathcal{R}\left(T^{n+1}\right)\right\}$, where the infimum over the empty set is taken to be infinity.

Theorem 2.5. Let $A, B \in \mathcal{B}_{R}(X)$ and let $q \in \mathbb{H} \backslash\{0\}$. Then

(i) $\operatorname{dsc}\left(\delta_{A, B}\right)=d s c\left(\delta_{B, A}\right)$;

(ii) $\operatorname{asc}\left(\delta_{A, B}\right)=\operatorname{asc}\left(\delta_{B, A}\right)$.

Proof. (i) Assume that $\mathcal{R}\left(\delta_{A, B}^{n}\right)=\mathcal{R}\left(\delta_{A, B}^{n+1}\right)$ for some $n \in \mathbb{N}$ and let $x \in X$. We have

$$
\begin{aligned}
& \delta_{B, A}^{n+1} x=\delta_{B, A}^{n} \delta_{B, A} x=\delta_{B, A}^{n}\left((B A)^{2} x-2 \operatorname{Re}(q) B A x+|q|^{2} x\right) \\
& =\delta_{B, A}^{n}(B A)^{2} x-2 \operatorname{Re}(q) \delta_{B, A}^{n} B A x+|q|^{2} \delta_{B, A}^{n} x .
\end{aligned}
$$

Since $\delta_{B, A} B=B \delta_{A, B}$,

$$
\delta_{B, A}^{n}(B A)^{2} x-2 \operatorname{Re}(q) \delta_{B, A}^{n} B A x+|q|^{2} \delta_{B, A}^{n} x=B \delta_{A, B}^{n} A B A x-2 \operatorname{Re}(q) B \delta_{A, B}^{n} A x+|q|^{2} \delta_{B, A}^{n} x .
$$

There exist $y, z \in X$ such that $\delta_{A, B}^{n} A B A x=\delta_{A, B}^{n+1} y$ and $\delta_{A, B}^{n} A x=\delta_{A, B}^{n+1} z$. Thus

$$
B \delta_{A, B}^{n} A B A x-2 \operatorname{Re}(q) B \delta_{A, B}^{n} A x+|q|^{2} \delta_{B, A}^{n} x=B \delta_{A, B}^{n+1} y-2 \operatorname{Re}(q) B \delta_{A, B}^{n+1} z+|q|^{2} \delta_{B, A}^{n} x .
$$

Therefore

$$
|q|^{2} \delta_{B, A}^{n} x=\delta_{B, A}^{n+1} x-\delta_{B, A}^{n+1} B y+2 \operatorname{Re}(q) \delta_{B, A}^{n+1} B z .
$$

Hence $\delta_{B, A}^{n} x \in \mathcal{R}\left(\delta_{B, A}^{n+1}\right)$, thus $\mathcal{R}\left(\delta_{B, A}^{n}\right)=\mathcal{R}\left(\delta_{B, A}^{n+1}\right)$. Then $\operatorname{dsc}\left(\delta_{B, A}\right) \leq d s c\left(\delta_{A, B}\right)$ and so

$$
\operatorname{dsc}\left(\delta_{B, A}\right)=\operatorname{dsc}\left(\delta_{A, B}\right) \text {. }
$$

(ii) Assume that $\mathcal{N}\left(\delta_{A, B}^{n}\right)=\mathcal{N}\left(\delta_{A, B}^{n+1}\right)$ for some $n \in \mathbb{N}$. Let $x \in X$ such that $\delta_{B, A}^{n+1} x=0$. We have

$$
\begin{aligned}
& \delta_{B, A}^{n+1} x=\delta_{B, A} \delta_{B, A}^{n} x=(B A)^{2} \delta_{B, A}^{n} x-2 \operatorname{Re}(q) B A \delta_{B, A}^{n} x+|q|^{2} \delta_{B, A}^{n} x \\
& =B A B \delta_{A, B}^{n} A x-2 \operatorname{Re}(q) B \delta_{A, B}^{n} A x+|q|^{2} \delta_{B, A}^{n} x .
\end{aligned}
$$

Since $0=A \delta_{B, A}^{n+1} x=\delta_{A, B}^{n+1} A x, A x \in \mathcal{N}\left(\delta_{A, B}^{n}\right)$. Then

$$
B A B \delta_{A, B}^{n} A x-2 \operatorname{Re}(q) B \delta_{A, B}^{n} A x+|q|^{2} \delta_{B, A}^{n} x=|q|^{2} \delta_{B, A}^{n} x=0 .
$$

Hence $x \in \mathcal{N}\left(\delta_{B, A}^{n}\right)$, thus $\mathcal{N}\left(\delta_{B, A}^{n}\right)=\mathcal{N}\left(\delta_{B, A}^{n+1}\right)$. Then $\operatorname{asc}\left(\delta_{B, A}\right) \leq \operatorname{asc}\left(\delta_{A, B}\right)$, and so

$$
\operatorname{asc}\left(\delta_{B, A}\right)=\operatorname{asc}\left(\delta_{A, B}\right) \text {. }
$$


Definition 2.1. Let $\mathcal{R}$ be a ring and let $a \in \mathcal{R}$. An element $b \in \mathcal{R}$ is a Drazin inverse of $a$, written $b=a^{d}$, if

$$
a b=b a, a b^{2}=b, a^{k+1} b=a^{k} .
$$

The least nonnegative integer $k$ for which these equations hold is the Drazin index $i(a)$ of $a$.

For a thorough treatment of the Drazin invertibility, the reader is referred, for instance, to $[1,7,9,13]$.

Lemma 2.2 ([7]). Let $\mathcal{R}$ be a ring and let $a \in \mathcal{R}$, then $a$ is Drazin invertible if and only if there exist $c, d \in \mathcal{R}$ and $n \in \mathbb{N}$ such that $a^{n+1} d=a^{n}=c a^{n+1}$, in this case, $i(a) \leq n$.

Theorem 2.6. Let $a, b \in \mathcal{A}, q \in \mathbb{H} \backslash\{0\}$. Then $\delta_{a, b}$ is Drazin invertible in $\mathcal{A}$ if and only if $\delta_{b, a}$ is. In such a case, $i\left(\delta_{a, b}\right)=i\left(\delta_{b, a}\right)$.

Proof. Assume that $\delta_{a, b}$ is Drazin invertible. Then $\delta_{a, b}^{n+1} z=\delta_{a, b}^{n}=z \delta_{a, b}^{n+1}$ where $z=\left(\delta_{a, b}\right)^{d}$ and $n:=i\left(\delta_{a, b}\right)$. Hence

$$
\delta_{b, a}^{n+1}(2 \operatorname{Re}(q) b z a-b z a b a+e)=2 \operatorname{Re}(q) \delta_{b, a}^{n+1} b z a-\delta_{b, a}^{n+1} b z a b a+\delta_{b, a}^{n+1} .
$$

Since $\delta_{b, a} b=b \delta_{a, b}$,

$$
\begin{aligned}
& 2 \operatorname{Re}(q) \delta_{b, a}^{n+1} b z a-\delta_{b, a}^{n+1} b z a b a+\delta_{b, a}^{n+1}=2 \operatorname{Re}(q) b \delta_{a, b}^{n+1} z a-b \delta_{a, b}^{n+1} z a b a+\delta_{b, a}^{n+1} \\
& =2 \operatorname{Re}(q) b \delta_{a, b}^{n} a-b \delta_{a, b}^{n} a b a+\delta_{b, a}^{n+1}=2 \operatorname{Re}(q) \delta_{b, a}^{n} b a-\delta_{b, a}^{n} b a b a+\delta_{b, a}^{n+1} \\
& =\delta_{b, a}^{n}\left(2 \operatorname{Re}(q) b a-b a b a+\delta_{b, a}\right)=|q|^{2} \delta_{b, a}^{n} .
\end{aligned}
$$

Similarly,

$$
(2 \operatorname{Re}(q) b z a-b a b z a+e) \delta_{b, a}^{n+1}=|q|^{2} \delta_{b, a}^{n} .
$$

By Lemma 2.2, $\delta_{b, a}$ is Drazin invertible and $i\left(\delta_{b, a}\right) \leq i\left(\delta_{a, b}\right)$.

If $\delta_{a, b}$ is Drazin invertible, then

$$
\left(\delta_{b, a}\right)^{d}=\frac{1}{|q|^{2}}\left(2 \operatorname{Re}(q) b\left(\delta_{a, b}\right)^{d} a-b\left(\delta_{a, b}\right)^{d} a b a+e\right) .
$$

Theorem 2.7. Let $a, b \in \mathcal{A}$. Suppose that $a b=e$ and $b a \neq e$. Then $\sigma_{S}(a)$ and $\sigma_{S}(b)$ contain $a$ neighbourhood of 0 .

Proof. For all $q \in \mathbb{H}$, we have

$$
\left(a^{2}-2 \operatorname{Re}(q) a+|q|^{2} e\right) b^{2}=e-2 \operatorname{Re}(q) b+|q|^{2} b^{2} .
$$

There exists $r>0$ such that for all $q \in\{q \in \mathbb{H}:|q|<r\}, e-2 \operatorname{Re}(q) b+|q|^{2} b^{2}$ is invertible (see [4, Lemma 3.1.12]). Then

$$
\left(a^{2}-2 \operatorname{Re}(q) a+|q|^{2} e\right) b^{2}\left(e-2 \operatorname{Re}(q) b+|q|^{2} b^{2}\right)^{-1}=e .
$$

Now suppose that $a^{2}-2 \operatorname{Re}(q) a+|q|^{2} e$ is invertible. Then

$$
b^{2}\left(e-2 \operatorname{Re}(q) b+|q|^{2} b^{2}\right)^{-1}\left(a^{2}-2 \operatorname{Re}(q) a+|q|^{2} e\right)=e .
$$


Since $b^{2}$ and $\left(e-2 \operatorname{Re}(q) b+|q|^{2} b^{2}\right)^{-1}$ commute,

$$
\left(e-2 \operatorname{Re}(q) b+|q|^{2} b^{2}\right)^{-1}\left(b^{2} a^{2}-2 \operatorname{Re}(q) b^{2} a+|q|^{2} b^{2}\right)=e,
$$

and so

$$
b^{2} a^{2}-2 \operatorname{Re}(q) b^{2} a+|q|^{2} b^{2}=e-2 \operatorname{Re}(q) b+|q|^{2} b^{2}
$$

Left multiplication by $a$ and right multiplication by $b$ show at once that

$$
b a=e
$$

which leads to a contradiction. And consequently $a^{2}-2 \operatorname{Re}(q) a+|q|^{2} e$ is not invertible. So we have proved that $\{q \in \mathbb{H}:|q|<r\} \subseteq \sigma_{S}(a)$. Similarly, $\sigma_{S}(b)$ contain a neighbourhood of 0 .

Remark 2.1. The theory we developed in this paper is also applies in the case of bounded left linear operators acting on a two-sided quaternionic Banach space with obvious modifications.

\section{Acknowledgments}

The authors would like to thank the referees for their very detailed comments and suggestions on a preliminary version of this manuscript.

\section{References}

[1] P. Aiena, M.T. Biondi and C. Carpintero, On Drazin invertibility, Proc. Amer. Math. Soc., 136 (2008), $2839-2848$.

[2] B. Aupetit, A primer on spectral theory, Springer Verlag, New York (1991).

[3] B.A. Barnes, Common operator properties of the linear operators RS and SR, Proc. Amer. Math. Soc., 126 (1998), 1055-1061.

[4] F. Colombo, J. Gantner and D.P. Kimsey, Spectral theory on the S-spectrum for quaternionic operators, Operator Theory: Advances and Applications, 270, Springer Basel (2019).

[5] F. Colombo, I. Sabadini and D.C. Struppa, Noncommutative Functional Calculus, Birkhäuser Basel (2011).

[6] D. Cvetkovi-Ili and R. Harte, On Jacobson's lemma and Drazin invertibility, Appl. Math. Letters, 23 (2010), 417-420.

[7] M.P. Drazin, Pseudo-inverses in associative rings and semigroups, Amer. Math. Monthly, 65 (1958), 506-514.

[8] R. Ghiloni, V. Moretti and A. Perotti, Continuous slice functional calculus in quaternionic Hilbert spaces, Rev. Math. Phys., 25 (2013) 1350006, 83 pp.

[9] C.F. King, A note on Drazin inverses, Pacific J. Math., 70 (1977), 383-390.

[10] T.E. Lam and P.P. Nielsen, Jacobsons lemma for Drazin inverses, in: Contemp. Math.: Ring theory and its applications, 609 (2014), 185-196.

[11] D. Mosi, Extensions of Jacobsons lemma for Drazin inverses, Aequationes Math., 91 (2017), 419-428.

[12] W. Rudin, Real and complex analysis, McGraw-Hill, New York (1986).

[13] Z. Wang, A class of Drazin inverses in rings, Filomat, 31 (2017), 1781-1789.

[14] K. Yan, Q. Zeng and Y. Zhu, Generalized Jacobson's lemma for Drazin inverses and its applications, Linear Multilinear Algebra, 68 (2020), 81-93. 